\title{
artigo
}

\section{Perfil e resultados perinatais de gestantes com síndrome hipertensiva do Sul do Brasil}

\author{
Profile and perinatal results of pregnant women with hypertensive syndrome in Southern Brazil \\ Perfil y resultados perinatales de mujeres embarazadas con síndrome hipertensivo en el Sur de Brasil
}

\begin{abstract}
RESUMO
Objetivou-se analisar o perfil e resultados perinatais de gestantes de alto risco com Síndrome Hipertensiva. Trata-se de um estudo epidemiológico, observacional e retrospectivo, com abordagem quantitativa. Foram utilizados dados secundários de 471 gestantes de alto risco e com Síndrome Hipertensiva da cidade de Sarandi/PR e posteriormente acompanhadas pelo ambulatório e realização do parto no hospital de referência, no período de 2012 a 2016, com partos realizados até 2017. As variáveis independentes foram constituídas pela Síndrome Hipertensiva, classificada em: hipertensão crônica, pré-eclâmpsia/eclâmpsia, pré-eclâmpsia sobreposta à hipertensão crônica, também conhecida como doença hipertensiva específica da gestação (DHEG). As variáveis desfechos foram: prematuridade, baixo peso ao nascer, Apgar $<7$ no $1^{\circ}$ e $5^{\circ}$ minutos, óbito fetal, óbito neonatal e parto cesárea. Os dados foram submetidos aos testes Qui-quadrado e Teste Exato de Fisher. Todas as discussões foram realizadas em nível de 5\% de significância $(p<0,05)$ e intervalo de confiança de $95 \%$. A Síndrome hipertensiva gestacional associa-se diretamente a prematuridade, 0 desenvolvimento de Apgar inferior a sete no quinto minuto de vida, o baixo peso ao nascer, a morte fetal e ao parto cesárea.
\end{abstract}

DESCRITORES: Saúde Materno-Infantil; Complicações na Gravidez; Hipertensão.

\section{ABSTRACT}

The aim was to analyze the profile and perinatal results of high-risk pregnant women with hypertensive syndrome. This is an epidemiological, observational and retrospective study, with a quantitative approach. Secondary data were used from 471 high-risk pregnant women with Hypertensive Syndrome in the city of Sarandi/PR and later followed by the outpatient clinic and delivery at the referral hospital, from 2012 to 2016, with deliveries performed until 2017. The independent variables were constituted by Hypertensive Syndrome, classified as: chronic hypertension, pre-eclampsia/eclampsia, pre-eclampsia overlapping with chronic hypertension, also known as hypertensive disease specific to pregnancy (DHEG). The outcome variables were prematurity, low birth weight, Apgar $<7$ in the 1st and 5th minutes, fetal death, neonatal death and cesarean delivery. The data were submitted to the Chi-square and Fisher's exact tests. All discussions were conducted at a $5 \%$ level of significance $(p<0.05)$ and a $95 \%$ confidence interval. Gestational hypertensive syndrome is directly associated with prematurity, the development of Apgar less than seven in the fifth minute of life, low birth weight, fetal death and cesarean delivery.

DESCRIPTORS: Maternal and Child Health; Pregnancy Complications; Hypertension.

\section{RESUMEN}

El objetivo fue analizar el perfil y los resultados perinatales de mujeres embarazadas de alto riesgo con síndrome hipertensivo. Este es un estudio epidemiológico, observacional y retrospectivo, con un enfoque cuantitativo. Se utilizaron datos secundarios de 471 mujeres embarazadas de alto riesgo con síndrome hipertensivo en la ciudad de Sarandi/PR y luego fueron seguidas por la clínica ambulatoria y el parto en el hospital de referencia, de 2012 a 2016, con partos realizados hasta 2017. Las variables independientes estaban constituidos por el Síndrome Hipertensivo, clasificado como: hipertensión crónica, preeclampsia/ eclampsia, preeclampsia solapada con hipertensión crónica, también conocida como enfermedad hipertensiva específica del embarazo (DHEG). Las variables de resultado fueron: prematuridad, bajo peso al nacer, Apgar $<7$ en el primer y quinto minutos, muerte fetal, muerte neonatal y parto por cesárea. Los datos fueron sometidos a las pruebas exactas de Chi-cuadrado y Fisher. Todas las discusiones se llevaron a cabo con un nivel de significación del $5 \%(p<0.05)$ y un intervalo de confianza del $95 \%$. El síndrome de hipertensión gestacional se asocia directamente con la prematuridad, el desarrollo de Apgar menos de siete en el quinto minuto de vida, bajo peso al nacer, muerte fetal y parto por cesárea.

DESCRIPTORES: Salud Maternoinfantil; Complicaciones del Embarazo; Hipertensión

RECEBIDO EM: 13/02/2020 APROVADO EM: 14/02/2020 


\section{Eduardo Marçal Garcia}

Acadêmico do Curso de Medicina do Centro Universitário de Maringá (UNICESUMAR), Maringá, Paraná, Brasil. https://orcid. org/0000-0001-9949-7341

\section{André Perez Candelorio}

Acadêmico do Curso de Medicina do Centro Universitário de Maringá (UNICESUMAR), Maringá, Paraná, Brasil. https://orcid. org/0000-0001-6034-1120

\section{Sandra Marisa Pelloso}

Doutora em Enfermagem pela Universidade de São Paulo (USP). Professora Titular da Universidade Estadual de Maringá (UEM), Maringá, Paraná, Brasil. https://orcid.org/0000-0001-8455-6839

\section{Marcos Benatti Antunes}

Doutor em Enfermagem pela Universidade Estadual de Maringá (UEM). Docente do Curso de Medicina do Centro Universitário de Maringá (UNICESUMAR), Maringá, Paraná, Brasil. https://orcid.org/0000-0002-5139-8827

\section{INTRODUÇÃO}

A Síndrome Hipertensiva (SH) gestacional vem ganhando destaque em debates sobre saúde no mundo, especialmente em países que buscam o desenvolvimento econômico e social, como o Brasil, e pode ser caracterizada por três tipos: hipertensão arterial crônica (HAS); hipertensão arterial crônica superposta por pré-eclâmpsia; e doença hipertensiva específica da gestação - $\mathrm{DHEG}^{(1,2)}$.

Dentre as frequentes complicações fetais advindas da $\mathrm{SH}$, destaca-se a morte perinatal, restrição de crescimento fetal e a prematuridade. Já as complicações maternas, verifica-se o descolamento prematuro de placenta, síndrome de HELLP, o desequilíbrio proteico em níveis séricos e uma expressiva diminuição da taxa de filtração glomerular $^{(3,4)}$.

O futuro neonato apresenta maior probabilidade a ser acometido por várias enfermidades, devido às consequências da $\mathrm{SH}$ gestacional. Estudos recentes apontam que gestantes com pré-eclâmpsia severa apresentam maiores chances de desenvolverem edema pulmonar, convulsões, insuficiência hepática, insuficiência renal aguda e coagulação intravascular disseminada ${ }^{(5)}$. Na hipertensão arterial crônica, a criança a termo apresenta um risco $50 \%$ maior do que o esperado para a idade gestacional, já o recém-nascido prematuro apresenta $450 \%$ de risco ampliado ${ }^{(6)}$.

Dados globais apontam que cerca de $3 \%$ a $8 \%$ das gestantes apresentam algum tipo de desordem relacionada ao descontrole da pressão arterial durante o processo de pré-natal, e, enquanto Organização Mundial da Saúde (OMS) estima que a referida enfermidade é responsável por cerca de $13 \%$ das mortes maternas no mundo. O Brasil possui uma realidade diferente, segundo dados do Ministério da Saúde (MS), aproxima-se de 35\%, com uma taxa de 140 a 160 mortes maternas para cada 100.000 nascidos vivos - $\mathrm{NV}^{(7,8)}$.

O distúrbio fisiológico e a taxa de mortalidade relativamente alta, fatores oriundos da SH gestacional, acarretam na queda da qualidade de vida da gestante em conjunto aos seus familiares, tocante ao constante medo de um possível primeiro aborto ou repetido aborto espontâneo, o qual pode ser desencadeado quando presente o quadro de hipertensão crônica ${ }^{(9)}$.

Além disso, o surgimento de $\mathrm{SH}$ de moderada ou grave intensidade apresenta-se com o dobro de incidência em mulheres que apresentam abortos espontâneos repetitivos, e, assim, faz-se possível constatar um aumento no fator de risco para o desenvolvimento de transtorno obsessivo-compulsivo (TOC) por parte dessas mulheres ${ }^{(10,11)}$.

Dessa forma, levando em consideração as políticas e programas públicos de atenção à gestante, a sublime incidência da $\mathrm{SH}$ na gestação, bem como a vastidão dos resultados desfavoráveis gestacionais e perinatais, o presente estudo objetivou analisar o perfil e resultados perinatais de gestantes de alto risco com $\mathrm{SH}$.

\section{METODOLOGIA}

Trata-se de um estudo epidemiológico, observacional e retrospectivo, com abordagem quantitativa.

O estudo foi realizado em um ambulatório de alto risco referência da $15^{\text {a }}$ Regional de Saúde (RS) da Região Noroeste do Paraná. Para a realização desse estudo, foram utilizados dados secundários (prontuários, relatórios, estratificação de risco e cartão do pré-natal) de 471 gestantes de alto risco e com $\mathrm{SH}$ da cidade de Sarandi/PR e, posteriormente, acompanhadas pelo ambulatório e realização do parto no hospital de referência, no período de 2012 a 2016, com partos realizados até 2017 . O processo de coleta de dados foi de novembro de 2016 a outubro de 2017.

As variáveis independentes foram constituídas pela SH, classificadas em: hipertensão crônica, pré-eclâmpsia/eclâmpsia, pré-eclâmpsia sobreposta à hipertensão crônica e hipertensão gestacional, também conhecida como doença hipertensiva específica da gestação (DHEG). De acordo com a estratificação de risco utilizada na região, as gestantes do presente estudo foram classificadas em: grupo I - gestantes com condição clínica preexistente de hipertensão arterial crônica; grupo II gestantes com antecedente obstétrico de pré-eclâmpsia; grupo III - gestantes com DHEG na gestação atual.

As variáveis desfechos foram: prematuridade, baixo peso ao nascer (BPN), Apgar 


\section{artigo}

Garcia, M.E.; Candelorio, A.P.; Pelloso, S.M.; Antunes, M.B.

Perfil e resultados perinatais de gestantes com sindrome hipertensiva do Sul do Brasil

$<7$ no $1^{\circ}$ e $5^{\circ}$ minutos, óbito fetal, óbito neonatal e parto cesárea.

Os critérios de inclusão para os grupos I, II e III foram: gestantes classificadas com SH que tiveram o parto no hospital de referência. Foram excluídas do estudo as gestantes que tiveram partos fora do estabelecimento de referência ao ambulatório de risco e que não apresentaram a $\mathrm{SH}$.
Para a compilação dos dados e análise estatística foi utilizado o software Epi Info, versão 7.0, programa de domínio público criado pelo Centers for Disease Control and Prevention (CDC). Os dados foram submetidos aos testes Qui-quadrado $\left(x^{2}\right)$ e Teste Exato de Fisher. Todas as discussões foram realizadas em nível de 5\% de significância

Tabela 1. Perfil sociodemográfico das gestantes estratificadas como alto risco. Sarandi, PR, Brasil, 2019.

$$
\text { Variáveis }
$$

$\mathrm{n}$

Idade

10 a 19

20 a 34

35 ou mais

Cor

Branca

Preta

Parda

Amarela

Situação conjugal

Vive com o companheiro

256

215

232

239

$\geq 8$ anos

Trabalho remunerado

Sim

262

209

$\%$

5,7

73,9

20,4

56,5

3,6

39,3

0,6

$03 \quad 0,6$

Não
49,3

54,4

45,6

50,7

55,6

44,4 $(\mathrm{p}<0,05)$ e intervalo de confiança (IC) de $95 \%$.

Foram atendidas todas as normas nacionais e internacionais de ética em pesquisa envolvendo seres humanos, sendo aprovado pelo parecer n. ${ }^{\circ}$ 2.287.476 do Comitê Permanente de Ética em Pesquisa com Seres Humanos da Universidade Estadual de Maringá (COPEP).

\section{RESULTADOS}

As 471 gestantes de alto risco deste estudo apresentaram como características predominantes: idade entre 20 e 34 anos ( $73,9 \%)$, pele branca ( $56,5 \%)$; vivem com o companheiro $(54,4 \%)$; escolaridade igual ou superior a 8 anos (50,7\%); e trabalho remunerado $(55,6 \%)$, conforme apresenta a Tabela 1.

A Tabela 2 apresenta a HAS como CCPE e a Pré-eclâmpsia como AO associadas ao Apgar reduzido no $5^{\circ}$ minuto $(\mathrm{p}=0,02)$ e ao parto cesárea $(\mathrm{p}<0,001)$. Além desses resultados, destaca-se o número de óbitos entre gestantes com SH. No Grupo I (HAS) houve 1,6\% de mortalidade fetal e 3,2\% de mortalidade infantil e no Grupo III (DHEG) houve 4,7\% de mortalidade infantil.

\section{DISCUSSÃO}

A Síndrome Hipertensiva Gestacional é uma doença que possui uma relevante taxa de incidência e prevalência

Tabela 2. Associação entre Síndrome Hipertensiva e resultados perinatais de gestação de alto risco, Sarandi, PR, Brasil, 2019.

Resultados Perinatais
Prematuridade (<37 semanas)
Baixo peso ao nascer (<2.500 g)
Apgar $1^{\circ}$ min. $(<7)$
Apgar $5^{\circ}$ min. $(<7)$
Morte fetal
Morte infantil
Cesárea

Grupo I

Hipertensão Arterial

$(\mathrm{n}=63)$

$\mathrm{n}$

27

15

15

08

01

02

56

$\%$

42,9

23,9

23,9

12,7

1,6

3,2

88,9
Grupo II

Pré-Eclâmpsia

$(\mathrm{n}=30)$

p

$P$
0,16

0,82

0,30

0,02

$0,51^{*}$

$0,23^{*}$

$<0,001$

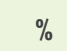

10

07

04

08

08

-

29

33,3

23,3
12,7
Grupo III

DHEG

$(\mathrm{n}=43)$

$\%$

34,9

P

0,84

15

13,9

0,15

0,93

25,6

0,25

4,7

$0,47^{*}$

0,02

02

-

\section{4,7}

$0,12^{*}$

79,1 
e, em nosso país, a mesma merece atenção devido aos diversos acometimentos originados a partir dela durante o ciclo gravídico, tanto para a mulher quanto para o feto, dentre esses acometimentos, pode-se encontrar: desenvolvimento de uma possível eclâmpsia, maior risco para síndrome de HELLP, partos prematuros, origem a bebês com baixo peso. Ademais, esta síndrome lidera hoje o ranking de doenças responsáveis por morte materna, principalmente quando se instala em suas formas mais graves ${ }^{(12)}$.

Dentro dos fatores abordados nesse estudo, encontra-se a idade gestacional tardia (acima dos 35 anos), com um total de 20,4\% mulheres. Nesse contexto, o fator etário é um agravante considerável quanto ao risco de hipertensão gestacional, pois mulheres acima de 35 anos apresentam maior susceptibilidade a comprometimento vascular. Além disso, mulheres que se encontram em idade avançada possuem fatores de risco para desenvolverem partos pré-termo e recém-nascidos com baixo peso ao nascer (BPN), o que resulta em bebês com baixos valores de Apgar ${ }^{(13,14)}$.

Outro aspecto retratado nesta pesquisa é a situação conjugal da gestante, algo que se mostra relevante para a análise dos dados. Nota-se que cerca de 45,6\% das participantes do estudo não vivem com o companheiro. Segundo alguns autores ${ }^{(15)}$, a situação conjugal instável é considerada um fator de risco para o desenvolvimento de agravos durante o processo gestacional, dentre esses agravos estão: BPN e aumento da mortalidade perinatal, isso pode ser explicado pelo fato da mulher precisar arcar com todas as responsabilidades no tocante à vida de seu filho, sem ter um parceiro para auxiliá-la.

Além disso, ter uma relação conjugal estável tem impacto direto no processo de gestação, uma vez que a mulher solitária apresenta maior fragilidade quanto a possíveis episódios de estresse, muitas vezes por terem que realizar atividades domésticas sem nenhum auxílio de um companheiro. Tais episódios podem provocar um descontrole da pressão arterial, tendo, assim, impacto indireto no

possível desenvolvimento de hipertensão gestacional ${ }^{(16)}$.

Ademais, o fator escolaridade foi analisado, onde pode-se observar a grande incidência de gestantes de risco com idade escolar inferior ou igual a 8 anos $(50,7 \%)$, dado esse que pode ser ocasionado pela gravidez precoce e/ou falta de compa-

Posto isso, e levando em consideração os resultados dessa pesquisa, observa-se que as gestantes acometidas pela Síndrome Hipertensiva

na gestação possuem filhos com maior vulnerabilidade de desenvolvimento de baixos valores de Apgar no quinto minuto $^{(1)}$.

nheiro, obrigando a mulher a abandonar a carreira acadêmica por precisar prover o sustento do próprio filho, o que ocorre porque grande parte das famílias não tem estrutura financeira suficiente para lidar com os futuros gastos da criança ${ }^{(17)}$.

Tal aspecto também foi abordado neste estudo, em que $55,6 \%$ das gestantes de risco já estão inseridas no mercado de trabalho. Além disso, segundo o Ministério da Saúde, a baixa escolaridade favorece a gestação de alto risco, por influenciar na escassez do aprendizado e do acesso à informação, tornando muitas vezes as consultas pré-natais pouco eficientes e/ou frequentadas, pelo motivo laboral ${ }^{(18)}$.

Nesse estudo, o Apgar $5^{\circ} \mathrm{min} .(<7)$ esteve relacionado com a Hipertensão Arterial e a Pré-Eclâmpsia, respectivamente com $(p=0,02)$, nesse sentido vale destacar, que o Apgar, é um instrumento muito utilizado em salas de parto, pois avalia as condições do recém-nascido dentro dos parâmetros da escala em questão, sendo que valores que se apresentam inferiores a sete no quinto minuto de vida da criança requerem intervenções médicas imediatas $^{(19)}$.

Posto isso, e levando em consideração os resultados dessa pesquisa, observa-se que as gestantes acometidas pela Síndrome Hipertensiva na gestação possuem filhos com maior vulnerabilidade de desenvolvimento de baixos valores de Apgar no quinto minuto ${ }^{(1)}$. Corroborando ainda, um estudo realizado com 368 gestantes com distúrbios hipertensivo, demonstrou que $14,9 \%$ do grupo deu origem a bebês com valor de Apgar inferior a sete entre o primeiro e quinto minuto de vida ${ }^{(20)}$.

Além disso, gestantes que possuem Síndrome Hipertensiva gestacional são susceptíveis e tem maiores chances de desenvolver fetos com policitemia que, por sua vez, se correlaciona com baixos valores de Apgar ${ }^{(21)}$. E ainda, o desenvolvimento de SH Gestacional possui relação direta com o grande número de morte materna e fetal, devido ao desenvolvimento de pré-eclâmpsia e a eclâmpsia, além de outra complicação na gestação ${ }^{(22)}$.

Dentro da suscetibilidade da gestante portadora de síndrome hipertensiva gestacional, existe a maior prevalência de cesarianas. Entretanto, o parto operatório possui diversos fatores de risco intrínsecos a ele, como o surgimento de sangramento intenso, o risco de contaminação por microrganismos e reações alérgicas aos anestésicos. Dessa maneira, o aumento de 


\section{artigo}

chance de surgimento de comorbidades no momento da concepção é evidente ${ }^{(4)}$.

Já em um estudo realizado com 108 mulheres que apresentaram pré-eclâmpsia devido ao aumento de pressão arterial, cerca de $68 \%$ das gestantes passaram por cesariana eletiva e $32 \%$ tentaram parto normal. Contudo, $26 \%$ das gestantes que gostariam de realizar o parto vaginal precisaram passar por cesariana de emergência por complicações diversas. Nesse contexto, a cesariana eletiva contribuiu para um melhor resultado perinatal do que o parto vaginal ou a cesariana de emergência após a indução do parto ${ }^{(23)}$.

\section{CONCLUSÃO}

A Síndrome hipertensiva gestacional associa-se diretamente com maiores riscos de desenvolvimento de diversas comorbidades durante o processo gestacional, dentre as quais destacam-se a prematuridade, o desenvolvimento de Apgar inferior a sete no quinto minuto de vida, o BPN, a morte fetal e o parto cesárea.
Com base no exposto, salienta-se a importância de uma equipe multidisciplinar preparada para proporcionar à gestante de risco um cuidado de qualidade e que seja efetivo, principalmente no processo de diagnóstico precoce, favorecendo, dessa maneira, o combate a possíveis intercorrências gestacionais futuras.

Vale reafirmar, ainda, a necessidade de um atendimento humanizado que avalie a gestante como um todo, abrangendo seus aspectos biopsicossociais, a fim de ter uma abordagem integral da paciente.

\section{REFERÊNCIAS}

1. Peraçoli JC, Parpinelli MA. Síndromes Hipertensivas da gestação: identificação de casos graves. Rev Bras Ginecol Obstet. 2005; 27(10):627-34.

2. Angonesi J, Polato A. Doença hipertensiva específica da gestação (DHEG), incidência à evolução para a Síndrome de HELLP. Rev. bras. anal. clin; 2007; 39(4):243-245.

3. Freire CMV, Tedoldi CL. Hipertensão arterial na gestação. Arquivos Brasileiros de Cardiologia, 2009; 93:159-65.

4. Lindheimer MD, Taler SJ, Cunningham FG. Hypertension in pregnancy. J Am Soc Hypertens. 2010; 4(2):68-78.

5. Sibai BM. Diagnosis and Management of Gestacional Hypertension and Preeclampsia. Obstet Gynecol. 2003; 102(1):18192.

6. Catov JM, Nohr EA, Olsen J et al. Chronic hypertension related to risk for preterm and term small for gestational age births. Obstet Gynecol. 2008; 112(2 Pt 1):290-6.

7. Moura MDR, Castro MP, Margotto PR et al. Hipertensão arterial na gestação - importância do seguimento materno no desfecho neonatal. Com. Ciências Saúde, 2011; 22 Sup 1:S113-S120.

8. Xiong $T, M u ~ Y$, Liang J et al. Hypertensive disorders in pregnancy and stillbirth rates: a facility-based study in China. 2018 Aug 1; 96(8):531-539.

9. Costa SHM, Ramos JGL, Vettori DV et al. Hipertensão crônica e complicações na gravidez. Revista da Sociedade de Cardiologia do Rio Grande do Sul, 2005; 5.

10. Geller PA, Klier CM, Neugebauer R. Anxiety disorders following miscarriage. J Clin Psychiatry. 2001; 62(6):432-8.

11. Francisco MFR, Mattar R, Bortoletti FF et al. Sexualidade e depressão em gestantes com aborto espontâneo de repetição. Rev. Bras. Ginecol. Obstet. 2014; 36(4):152-156.

12. Aguiar MIF, Freire PBG, Cruz IMP, et al. Sistematização da Assistência de Enfermagem a Paciente com Síndrome Hipertensiva Específica da Gestação. Revista da Rede de Enfermagem do Nordeste, 2010; 11(4):66-75.
13. Alves NCC, Feitosa KMA, Mendes MES et al. Complicações na gestação em mulheres com idade maior ou igual a 35 anos. Rev. Gaúcha Enferm. 2017; 38(4):e2017-0042.

14. Pinheiro RL, Areia AL, Mota Pinto $A$ et al. Idade Materna Avançada: Desfechos Adversos da Gravidez, Uma Meta-Análise. Acta Médica Portuguesa, 2019; 32(3):219-226.

15. Rezende CL, Souza JC. Qualidade de vida das gestantes de alto risco de um centro de atendimento à mulher. Psicol. inf. 2012; 16(16):45-69.

16. Monteiro PGA, Souza IC, Rodrigues VCC et al. Percepções de mulheres acerca do estresse vivenciado na gestação. Investigação Qualitativa em Saúde. 2018; (2):1142-49.

17. Stefamo DD, Moccellin AS, Fabbro MRC et al. Caracterização das condições sociais e de saúde de mães adolescentes no primeiro ano pós-parto em um município do estado de São Paulo. Revista Baiana de Saúde Pública. 2011; 35(4):795-812.

18. Melo WA, Alves Jl, Ferreira AAS et al. Gestação de alto risco: fatores associados em município do Noroeste paranaense. Revista de Saúde Pública do Paraná. 2015; 17(1):82-91.

19. Antunes MB, Demitto MO, Gravena AAF et al. Síndrome hipertensiva e resultados perinatais em gestação de alto risco. Rev. Min. Enferm. 2017; 21:e-1057.

20. Adu-bonsaffoh K, Ntumy MY, Obed SA et al. Perinatal outcomes of hypertensive disorders in pregnancy at a tertiary hospital in Ghana. BMC Pregnancy Chirldbirth, 2017; 17(1):388.

21. Okoye HC, Nwogoh B, Odetunde OI. Correlation of hematocrit and Apgar scores in newborns of women with hypertensive disorders in pregnancy. Journal of Neonatal-Perinatal Medicine, 2017; 10(4):387-392.

22. Un Nisa S, Shaikh AA, Kumar R. Maternal and Fetal Outcomes of Pregnancy-related Hypertensive Disorders in a Tertiary Care Hospital in Sukkur, Pakistan. Cureus. 2019; 11(8):e5507.

23. Mashiloane CD, Moodley J. Induction or caesarean section for preterm pre-eclampsia? Journal of Obstetrics and Gynaecology. 2002; 22(4):353-356. 